Article

\title{
Cold-Sprayed Al6061 Coatings: Online Spray Monitoring and Influence of Process Parameters on Coating Properties
}

\author{
Heli Koivuluoto ${ }^{1, *(\mathbb{D}}$, Jussi Larjo ${ }^{2}$, Danilo Marini ${ }^{3}$, Giovanni Pulci ${ }^{3}\left[\right.$ and Francesco Marra $^{3}(\mathbb{C})$ \\ 1 Materials Science and Environmental Engineering, Faculty of Engineering and Natural Sciences, Tampere \\ University, 33100 Tampere, Finland \\ 2 Oseir Ltd., 33720 Tampere, Finland; jussi.larjo@oseir.com \\ 3 INSTM Reference laboratory for Engineering of Surface Treatment, Department of Chemical Engineering \\ Materials Environment, Sapienza University of Rome, 00185 Rome, Italy; danilo.marini@uniroma1.it (D.M.); \\ giovanni.pulci@uniroma1.it (G.P.); francesco.marra@uniroma1.it (F.M.) \\ * Correspondence: heli.koivuluoto@tuni.fi; Tel.: +358-40-849-0188
}

Received: 25 February 2020; Accepted: 1 April 2020; Published: 3 April 2020

\begin{abstract}
Process optimization and quality control are important issues in cold spraying and coating development. Because the cold spray processing is based on high kinetic energy by high particle velocities, online spray monitoring of particle inflight properties can be used as an assisting process tool. Particle velocities, their positions in the spray jet, and particle size measurements give valuable information about spraying conditions. This, in turn, improves reproducibility and reliability of coating production. This study focuses on cold spraying of Al6061 material and the connections between particle inflight properties and coating characteristics such as structures and mechanical properties. Furthermore, novel 2D velocity scan maps done with the HW CS2 online spray monitoring system are presented as an advantageous powder and spray condition controlling tool. Cold spray processing conditions were similar using different process parameters, confirmed with the online spray monitoring prior to coating production. Higher particle velocities led to higher particle deformation and thus, higher coating quality, denser structures, and improved adhesions. Also, deposition efficiency increased significantly by using higher particle velocities.
\end{abstract}

Keywords: cold spray; diagnostics; aluminum; Al6061; coatings; online monitoring; process parameters

\section{Introduction}

Cold spraying has shown its potential for producing high-quality coatings with different industrial requirements. For example, corrosion resistance, electrical conductivity, wear resistance, functional properties, and repairing possibilities are application fields for cold-sprayed coatings. Especially, for corrosion protection and repairing possibilities, $\mathrm{Al}$ and $\mathrm{Al}$-based alloy coatings have received a lot of interest due to their good material properties and high suitability for cold spraying as the feedstock materials [1-5]. In cold spraying, low melting point materials such as $\mathrm{Al}$ alloys can be sprayed without oxidation. This is because the cold spraying is the solid-state coating processing method, where coating formation is based on a high kinetic energy instead of thermal energy compared to other thermal spray technologies, e.g., arc and flame spray processes. Powder particles are accelerated with a high pressurized and preheated gas $\left(\mathrm{N}_{2}, \mathrm{He}\right.$, air) to a high velocity and they impact to a substrate surface, adhere, deform, and form a coating. [6-11] During the impacts, particles need to undergo a high plastic deformation in order to achieve high-quality coating structures. This, in turn, depends on the particle velocity. [12] Assadi et al. [12] have well summarized the influences of several factors 
on the particle velocity: (1) particle velocity is higher while spraying with He compared to $\mathrm{N}_{2}$; (2) particle velocity increases with increased gas temperature; (3) higher pressure leads to higher particle velocity; (4) particle velocity can be increased with certain nozzle geometries such as when length, expansion ratio or diameter increase, particle velocity increases; (5) by increasing the spray distance to the certain maximum level, particle velocity increases and then, after maximum distance decreases; (6) particle velocity decreases if spraying angle increases; and (7) if powder feeding rate increases, particle velocity decreases.

As noted, cold spray processing is influenced by several factors and they have combined effects. The process itself (low-, medium-, or high-pressure cold spray process) affects spray parameter selections, which can be used for spraying of different powder materials. These spray parameters have affected the particle inflight properties and powder deformation as well as thermal softening and adiabatic shear instabilities of impacted particles. [6-10] All factors should be taken into account while selecting the process parameters. In addition to this, powder and its properties can affect the sprayability and the deform capability during the processing as well as the spray parameter selection. Particles need to achieve certain material-dependent velocities in order to form a coating. Below the critical velocity, particles just rebound and do not adhere to the sprayed surface. Also, there is an upper velocity limit and above that, erosion starts to play a role and the number of bonded particles decreases. [12-14] Yin at al. [15] have reviewed the gas and particle interactions in cold spraying. Particle velocities has been modelled by using computational fluid dynamics (CFD) models $[14,16]$ and experimentally investigated by using different measuring techniques such as laser-2-focus (L2F) [17], doppler picture velocimetry (DPV) [18-21], and particle imaging velocimetry (PIV) [22-25]. Also, we have demonstrated the advanced optical diagnostic method called PTV (particle tracking velocimetry) or S-PTV (sizing-particle tracking velocimetry) [26], which is based on the PIV approach. In this measurement technique, more accurate results can be investigated due to its specific telecentric optics and advance measuring algorithm. Here, individual particle velocities, particle size, and particle position in the spray jet can be analyzed and used for process optimization and the spray parameter selection. Furthermore, the process quality and especially, powder travelling properties can be controlled. Online spray monitoring can indicate if any feeding variables or nozzle condition changes occur. In addition to particle velocity measurement with diagnostic measuring devices, powder jet has been investigated with an infra-red camera [27]. Its purpose is to observe integral particle stream properties rather than properties of individual particles in cold spraying. Therefore, it can give just an additional information about the powder jet.

Mean particle velocities have been reported in many studies [17,19,20,28]. Gilmore et al. [17] have investigated mean particle velocities and separate velocities of $\mathrm{Cu}$ particles by using the L2F technique, resulting in the smaller particles having higher velocities. Jodoin et al. [28] have studied cold spray particle velocities by modeling and connecting those particle velocities to experimental measurements with the PIV technique. They reported higher particle velocities with higher pressures and temperature used. Measured mean particle velocities were slightly higher or close to velocities modelled with CFD. In addition, shock waves of gas flow in the nozzle have been modelled, showing the dropping of the particle velocity before nozzle exit. This shock wave behavior cannot be detected with online monitoring techniques, which indicated that different analyzing techniques are supporting each other $[18,20,28]$. In addition, Silvello et al. [29] have analyzed several studies and depicted a process parameter, which has been described as material density divided by particle diameter times gas pressure per gas density. They have connected this to particle velocities. Particle velocity increased with increasing process parameter until reaching a certain point and then started to decrease. Also, particle velocity has been influenced by particle shape. Fukunuma et al. [19] have reported that irregular particles had higher average particle velocities compared with spherical particles.

Aluminum alloys are relatively easy to spray with cold spraying, but also present challenges caused by nozzle clogging, powder agglomeration during feeding, and uneven feeding. Therefore, process controlling, and quality checking are very important and advantageous in order to avoid 
the problems during the spray processing. We focus on aluminum alloy Al6061 because it has high corrosion resistance and good mechanical properties and thus, it is used in many common working conditions, e.g., in aircraft and automotive industries. Al6061 is a precipitation-hardened Al-based alloy, which has $\mathrm{Mg}$ and $\mathrm{Si}$ as major alloying elements [30]. Earlier, we achieved improvements in coating quality and properties by using laser-assisted cold spraying compared to traditional medium-pressure cold spraying [2]. Structure was denser and adhesion was higher together with higher deposition efficiency. In this study, we use a high-pressure cold spray system with advanced processing conditions for producing Al6061 coatings on Al and steel substrates.

This study focuses on the optimization of cold spraying of Al6061 by using novel online spray monitoring for the spray parameter selection and as the quality control tool. From the online monitoring point of view, particle velocities, particle size, and particle positions in the gas flow or gas jet are the factors that have been measured and used for the process optimization. There are multiple factors that influence the spray process, and they are summarized here. Particle-gas flow has specific features depending on the cold spray system used (e.g., high pressure vs. low pressure and axial feeding vs. radial feeding), process gas $\left(\mathrm{N}_{2}, \mathrm{He}\right.$, air), and nozzle design, geometry, and shape (e.g., round vs. rectangular shape), as well as process conditions (pressure, temperature, powder feeding) and powder properties (melting point, particle size distribution, shape and flowability). Also, these different factors influence mutually in the cold spray process. Therefore, it is crucial to develop the quality control aspects and methods together with the process development itself. This way, repeatability can be checked and at the same time, fast notification about cold spray equipment conditions can be achieved. For example, problems with uneven powder feeding, starting of nozzle clogging or wearing of the nozzle can be detected with the online spray monitoring. Importantly, fast diagnostics of powder conditions can be analyzed with particle size measurements. Sometimes, altered powders can lose their optimal properties during storage and this can be detected as particle size variations between different spray times. Particle inflight properties directly affect the coating properties and quality, and therefore, it is encouraged to study these properly during the cold spray process.

Online spray monitoring was done with a laser illuminated PTV as a multi-streak detection. In this study, a sheet illumination was used with HiWatch CS2 (HW CS2) online spray monitoring equipment. Furthermore, in this study, a 2D model for particle velocity data with particle position and amounts is presented. This trigging is shown first time here and its benefits are related to showing velocity distribution and cross-dependencies. After online spray monitoring, coating properties of cold-sprayed Al6061 coatings, and effect of spray parameters on those are analyzed and connected to particle velocities.

\section{Materials and Methods}

Aluminum alloy powder, A16061, was used as a feedstock material (A16061, TLS Technik, Bitterfeld-Wolfen, Germany). Composition is $\mathrm{Al}$ (bal-wt \%), Si 0.40-0.80 wt \%, Fe max. $0.7 \mathrm{wt} \%$, Cu 0.15-0.40 wt \%, Mn 0.15 wt \%, Mg 0.8-1.2 wt \%, Cr 0.04-0.35 wt \%, Zn max 0.25 wt \%, and Ti max. $0.15 \mathrm{wt} \%$. The powder was produced by gas-atomization for cold spraying, and it had a particle size distribution of $-40+10 \mu \mathrm{m}$. Particles were spherical in shape, Figure 1.

Coatings were manufactured with high-pressure cold spray equipment, PCS-100 (Plasma Giken Co., Ltd., Saitama, Japan). Nitrogen was used as a process gas. Spray parameters were selected as following: pressures of $30-40 \mathrm{bar}$, temperatures of $300-400{ }^{\circ} \mathrm{C}$, spray distance of $40 \mathrm{~mm}$, step size of $1 \mathrm{~mm}$, traverse speed of 10-20 m/min, powder feed of $1.5 \mathrm{rpm}$ (roughly measured as $\sim 10 \mathrm{~g} / \mathrm{min}$ ), and 4 coating layers. Converging-diverging de Laval nozzle with the outer diameter of $28 \mathrm{~mm}$ was used in these experiments. The nozzle was plastic, having a round shape. Coatings were sprayed using a robot (IRB 4600, ABB Ltd., Helsinki, Finland). Grit-blasted $\left(\mathrm{Al}_{2} \mathrm{O}_{3}\right.$, Mesh40) aluminum and low-carbon steel were used as substrates. Table 1 shows the test conditions used in this research for the online spray monitoring as well as for the coating production. 


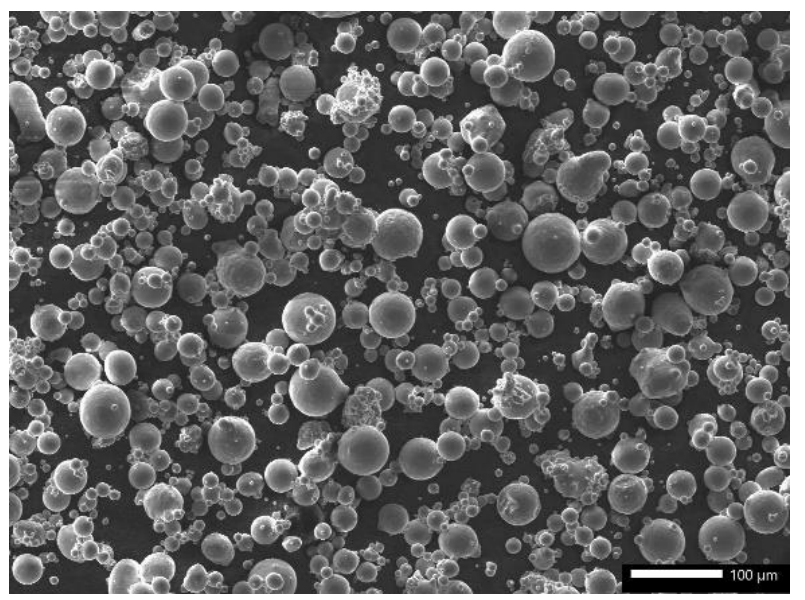

Figure 1. Morphology of gas-atomized Al6061 powder. SEM image.

Table 1. Cold spray test conditions and prepared coatings.

\begin{tabular}{ccccc}
\hline Test & Pressure (bar) & Temperature $\left({ }^{\circ} \mathbf{C}\right)$ & Online Spray Monitoring & Coating Code \\
\hline 1 & 30 & 300 & $\mathrm{X}$ & - \\
2 & 30 & 350 & $\mathrm{X}$ & - \\
3 & 30 & 400 & $\mathrm{X}$ & $\mathrm{Al}_{30} 400$ \\
4 & 35 & 300 & $\mathrm{X}$ & $\mathrm{Al}_{35} 300$ \\
5 & 35 & 350 & $\mathrm{X}$ & $\mathrm{Al}_{35} 350$ \\
6 & 35 & 400 & $\mathrm{X}$ & $\mathrm{Al}_{35} 400$ \\
7 & 40 & 300 & $\mathrm{X}$ & - \\
8 & 40 & 350 & $\mathrm{X}$ & - \\
9 & 40 & 400 & & $\mathrm{Al}_{40} 400$ \\
\hline
\end{tabular}

Novel online spray monitoring technique, PTV, (HiWatch CS2, HW CS2, Oseir Ltd., Tampere, Finland) was used prior to spraying the coating samples. Particle velocity, position, and size were measured from the mid-point (area $8 \times 5 \mathrm{~mm}^{2}, 2 \mathrm{MP}, 35 \mathrm{fps}$ (GigE)). In our previous study [26], we used HW HR2 (Oseir Ltd., Tampere, Finland) online spray monitoring system, which detects similar variables as HW CS2 used in this study. HW HR2 bases on shadow imaging whereas HW CS2 monitoring is based on a sheet measurement, where particles are detected by scattering. Both systems are based on imaging optical setup from narrow axial cross section of the spray and pulsed diode laser illumination of particles.

Compared to the more advanced S-PTV technique used earlier [26], this simple PTV technique has only coarse particle sizing, but it offers reduced system size and cost with much higher particle measurement rate. In both cases, the illumination and individual particle detection are based on a triple-pulse scheme [26]. The pulse interval in the triplet was $400 \mathrm{~ns}$. The camera (Figure 2a) itself is lightweight and simple to use. Figure $2 \mathrm{~b}$ shows particle imaging geometry used in the measurements. Details of the measurement details setup can be found from [26]. Single illuminated image covers a spray jet cross section of $\left(0.5 \times 5 \mathrm{~mm}^{2}\right)$. For this reason, precise alignment of spray nozzle with the imaging plane is needed. The camera control software provides automated image processing and spray particle position and velocity measurement, and also calculates mean values, distributions, cross-plots, and history graphs for various particle statistics. In addition, 2D spray cross section mapping was done (Figure 2c). The sheet translation was done with triggered burst imaging, where the sheet traverses the spray plume during image sequence capture. A typical image sequence is captured using $1 \mathrm{~mm} / \mathrm{s}$ scan speed producing 250 individual images (capture time $\sim 10 \mathrm{~s}$ ), thus providing a depth resolution of $0.04 \mathrm{~mm}$. Total analysis time is $\sim 15 \mathrm{~s}$. The resulting particle data table can be converted to a 2D map of particle properties describing the particle jet velocity and density distributions across the measured 
jet cross section. 2D velocity and density scan maps were measured with total cross section areas of $8 \times 8 \mathrm{~mm}^{2}$.

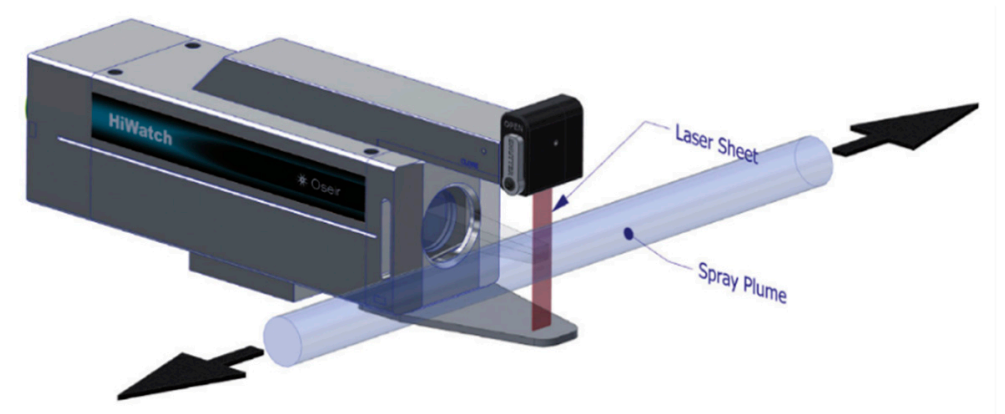

(a)

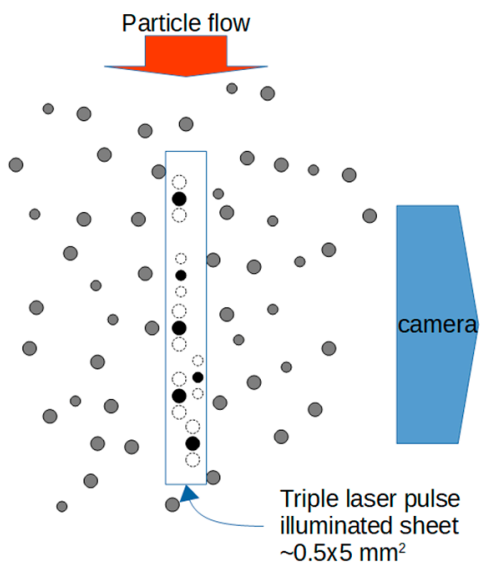

(b)

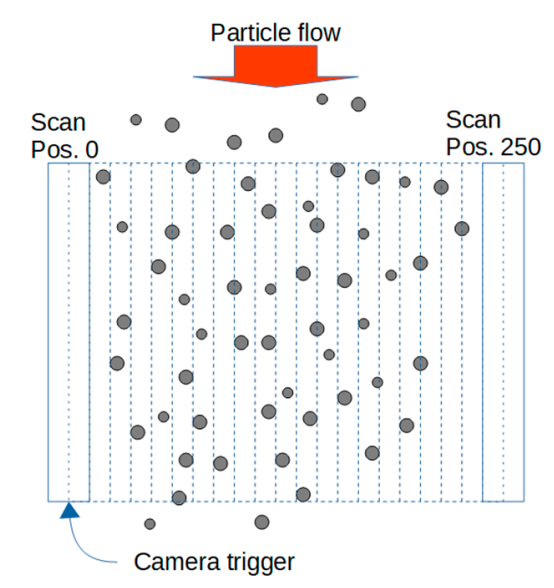

(c)

Figure 2. Online spray monitoring (a) HW CS2 system, (b) particle imaging geometry, and (c) scanning plume for 2D mapping.

Powder morphology was analyzed with a scanning electron microscope (SEM, Jeol IT-500, Tokyo, Japan). Coating structures were, in turn, studied with an optical light microscope (LM, Leica DM 2500, Wetzlar, Germany) and a field-emission scanning electron microscope (FESEM, Zeiss UltraPlus, Jena, Germany). Cross sections of the coatings were done following a standard sample preparation method, including polishing steps with SiC papers (up to 2500), diamond suspensions ( 3 and $1 \mu \mathrm{m}$ ), and final polishing with $\mathrm{SiO}_{2}$ suspension. Vickers hardness was measured with a Vickers hardness tester (Matsuzawa MMT-X7, Akita, Japan) by using the load of $100 \mathrm{~g}$. The results are given as an average of ten measurements. Coating thicknesses were measured from the coating cross sections pictured with LM and the results are given as an average of ten measurements.

Adhesion strength of coatings was measured through a mechanical testing machine (Instron 5584, Norwood, MA, USA) at a crosshead rate of $1 \mathrm{~mm} / \mathrm{min}$ according to the ASTM C633 [31]. Each test specimen was obtained assembling one cylindrical coated substrate to the sand-blasted faces of the loading fixtures by an adhesive bonding agent (Polyamide-epoxy FM 1000 Adhesive Film, bond strength $\sim 70 \mathrm{MPa}$ ); a self-aligning device was adopted for applying the tensile load to the assembly of the coating and fixtures.

\section{Results and Discussion}

Firstly, this study focuses on online spray monitoring, and secondly, coating properties sprayed with the selected spray parameters. Particle velocity and size as well as velocity distribution together with particle densities and positions are evaluated and followed with the characterization of the coating properties such as structure, thickness, hardness, and adhesion. 


\subsection{Particle Velocity Measurements}

Firstly, we present what kind of results can be collected from the measurement data with the optical diagnostic system, HW CS2, used in this study. Examples of the particle velocity distribution, particle size distribution, particle velocity distribution versus particle position in the spray jet, and statistics of the data measured with HW CS2 system are presented in Figure 3. This shows that many variables can be evaluated simultaneously.

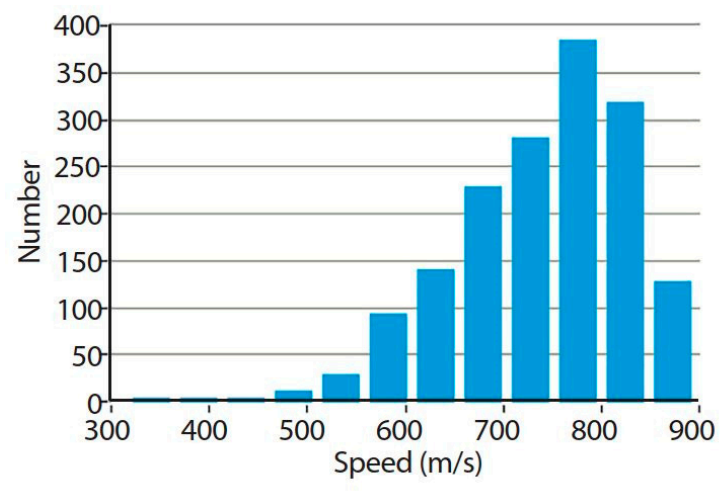

(a)

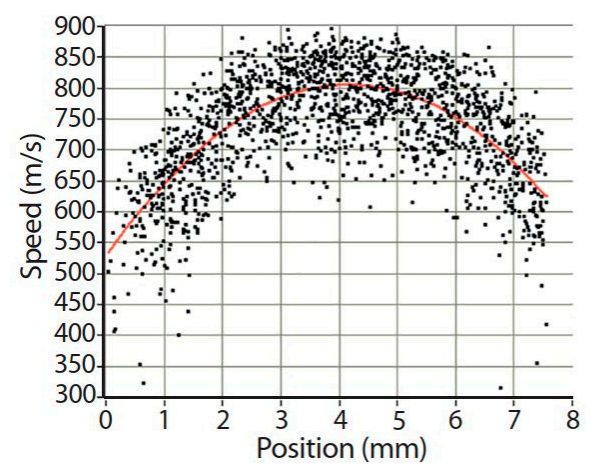

(c)

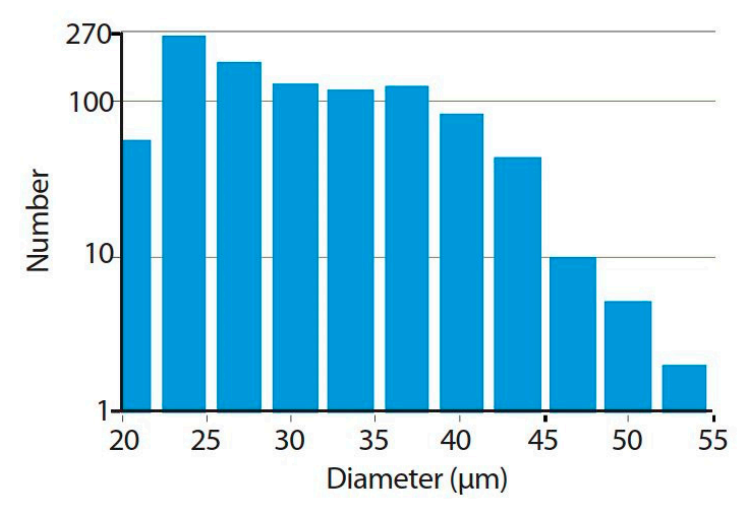

(b)

(d)

Figure 3. Example of the HW CS2 measurement data: (a) particle speed distribution, (b) particle size distribution, (c) particle speed distribution in x-position with fitting, and (d) statistics from the measured data.

In this study, particle velocities sprayed with nine totally different combinations of pressures (low: 30 bar, medium: 35 bar, and high: 40 bar) and temperatures (low: $300{ }^{\circ} \mathrm{C}$, medium: $350{ }^{\circ} \mathrm{C}$, and high: $400{ }^{\circ} \mathrm{C}$ ) were measured. The particle velocities as a function of pressure and temperature are presented in Figure 4 . When pressure and temperature increase, particle velocity increases as stated in many studies [12,13,25,26,32]. In the present study, temperature has a higher influence on particle velocity compared to pressure while spraying the Al6061 powder. Especially, when spraying with the highest temperature $\left(400^{\circ} \mathrm{C}\right)$ and the lowest pressure, particle velocities are almost at the similar level while spraying with the lowest temperature and the highest pressure. Increment of the particle velocities with medium or high pressure compared to low pressure is $4 \%$ and $6 \%$, respectively. Similarity spraying with the highest pressure (40 bar) increment of the particle velocity with medium or high temperature compared to low temperature is $2 \%$ and $5 \%$, respectively. The highest difference in the average particle velocities is between the lowest $\left(30 \mathrm{bar}, 300{ }^{\circ} \mathrm{C}\right)$ and the highest parameters 
$\left(40 \mathrm{bar}, 400^{\circ} \mathrm{C}\right)$ used. Particle velocities sprayed with the highest parameters were $10.5 \%$ higher than sprayed with the lowest parameters.

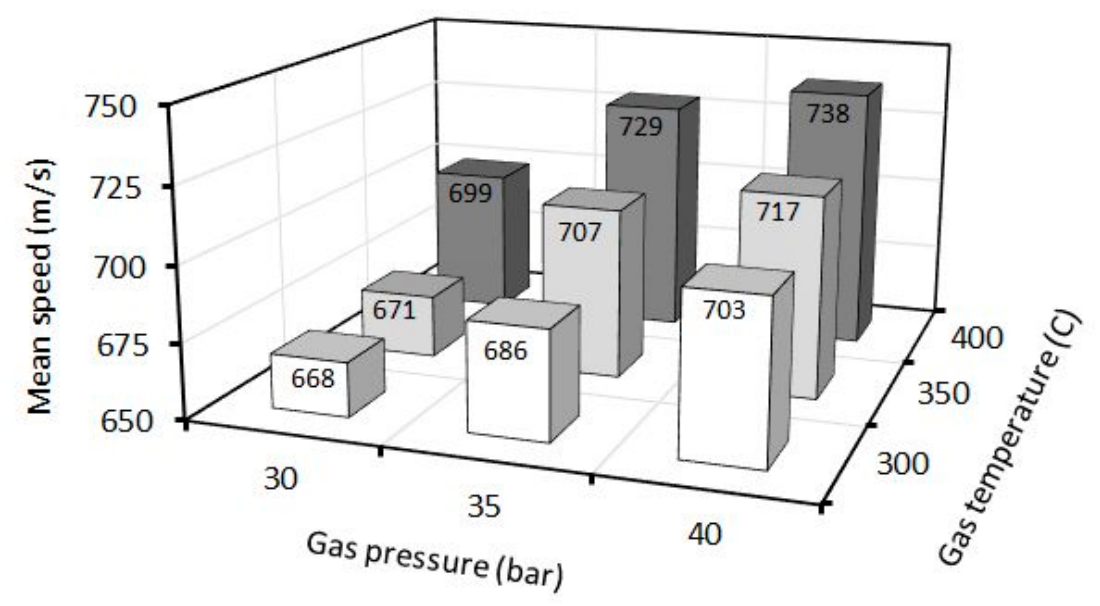

Figure 4. Particle mean velocities sprayed with different gas pressures and gas temperatures.

Meyer et al. [25] have measured particle velocities for Al with the PIV technique and the velocity dependence on the particle mass feed rates, resulting in decreased particle velocities with higher feed rates. Aluminum particles sprayed with the pressure of 15 bar or 30 bar had average particle velocities of $\sim 450$ and $\sim 540 \mathrm{~m} / \mathrm{s}$, respectively [25], using similar feed rates as we have used. In our study, particle velocities are higher, probably due to the higher gas temperatures and pressures used, differences in particle size and different particle velocity measuring technique used (PIV vs. PTV). Schmidt et al. [13] have shown critical velocities around $620-660 \mathrm{~m} / \mathrm{s}$ for $\mathrm{Al}$ and its alloys. In this study, all measured velocity values are above this, and therefore, coating formed successfully with all the selected spray parameters.

In addition to traditional particle velocity measurements, 2D velocity map was measured and analyzed. This novel measurement setup shows the deviation of particle positions and particle velocities in the spray plume from the cross-sectional direction. Particle concentration in different $x$-positions in the spray jet as well as particle velocity range in z-positions. Figure $5 a-c$ shows the $2 \mathrm{D}$ velocity scan maps for different gas pressures $\left(30,35\right.$, and 40 bar) used with the temperature of $400{ }^{\circ} \mathrm{C}$, respectively. It is clearly seen that velocity range increased with increased pressure. Additionally, particle positions in cross-sectional area in the spray plume can be detected. This is an advantageous quality control tool to check the nozzle and powder feeding conditions during the measurements. The measurements were achieved without substrate, and therefore, bow shock effect was not analyzed. However, bow shocks formed close to substrate surface has shown to affect particle impact velocity [15]. High-pressure and high-density gas generated this bow shock layer, which decreases particle velocity: this influence is stronger with small particles [15,33]. In this study, spray conditions were suitable, confirmed with these $2 \mathrm{D}$ velocity scan maps. $2 \mathrm{D}$ velocity scan maps showed that the highest velocities are in the middle area of the spray jet in $x$ and $z$ directions. Similarity, Meyer et al. [25] have shown that particle velocities of $\mathrm{Al}$ powder was the highest in the middle of the spray jet in $\mathrm{x}$ and directions. 


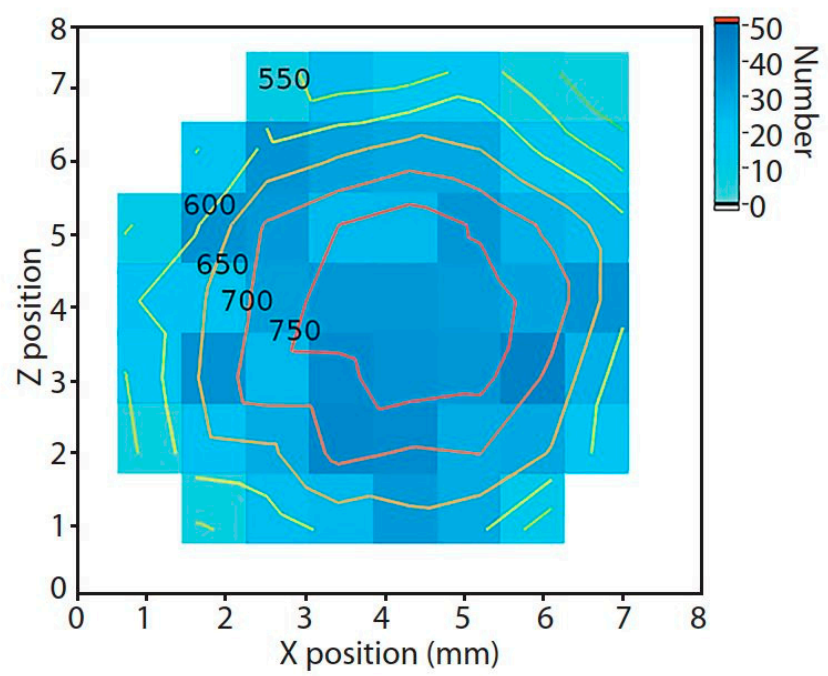

(a)

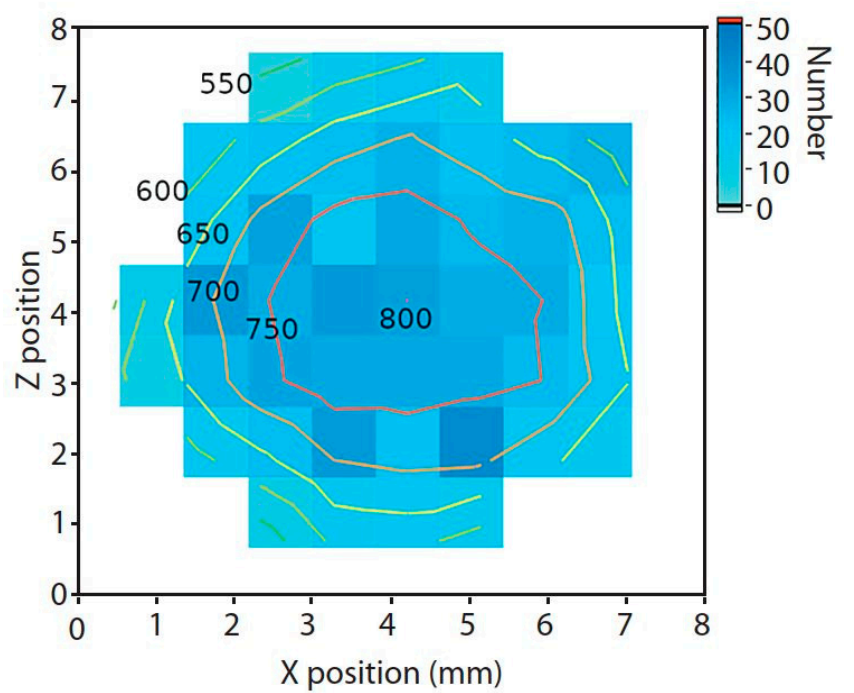

(b)

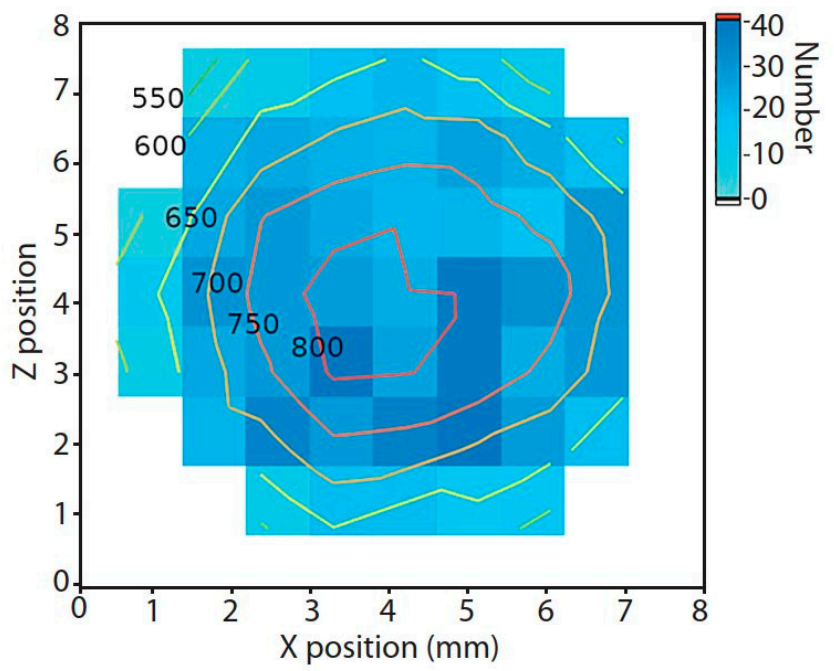

(c)

Figure 5. 2D velocity scan maps. Cold spraying of Al6061 powder with (a) 30 bar and $400{ }^{\circ} \mathrm{C}$, (b) 35 bar and $400{ }^{\circ} \mathrm{C}$, and (c) 40 bar and $400{ }^{\circ} \mathrm{C}$. Contour lines show velocity. Colored maps present particle densities. Scan area is $8 \times 8 \mathrm{~mm}^{2}$. 


\subsection{Particle Size Measurements}

The PTV technique in HW CS2 enables measuring particle sizes for quality inspection. This method analyzes particle sizes above $20 \mu \mathrm{m}$ for calculations but detects smaller sizes as well. It was a suitable monitoring tool for our study to verify the spray conditions and control powder quality. If particle size is changing, it could indicate agglomeration or some sticking during the powder feeding and spraying. In addition, one advantage of measuring particle sizes with diagnostics is that an amount of the measured particles is much higher compared to measurements done by image analysis from microscopic samples or with laser diffraction particle size measuring method [26]. In this study, particle sizes were in a similar level, indicating uniform powder feeding and powder conditions between several measurements and spraying. Particle position was measured by measuring the area and particles $/ \mathrm{mm}^{2}$, and the particle sizes where analyzed from this data. Table 2 shows the particle mean size, DV50 (cumulative 50\% particle size; half of the particles have the size below this value and the half above this value), and DV90 (cumulative $90 \%$ particle size; $90 \%$ of particles have the size below this value) values when spraying the powder with the pressure of 35 bar and different temperatures $\left(300,350\right.$, and $\left.400^{\circ} \mathrm{C}\right)$. The maximum difference in the particle sizes sprayed with different parameters is $0.9 \mu \mathrm{m}$, which is kept very low by taking into account deviation coming from the particle size distribution.

Table 2. Particle sizes measured with HW CS2 using pressure of 35 bar and gas temperatures of $300-400{ }^{\circ} \mathrm{C}$. Mean size, DV50, and DV90 values are presented.

\begin{tabular}{cccccc}
\hline Pressure (bar) & Temperature $\left({ }^{\circ} \mathbf{C}\right)$ & Mean Speed $(\mathrm{m} / \mathbf{s})$ & Mean Size $(\mu \mathrm{m})$ & DV50 $(\mu \mathrm{m})$ & DV90 $(\mu \mathrm{m})$ \\
\hline 35 & 300 & 686 & 29.5 & 35.2 & 45.6 \\
35 & 350 & 707 & 30.1 & 35.5 & 43.4 \\
35 & 400 & 729 & 30.4 & 36.4 & 43.6 \\
\hline
\end{tabular}

It is important to measure the particle size together with particle velocities. Additionally, particle velocity is depended on the particle size; smaller particles are faster and the larger ones opposite are slower [13]. Also, as noted earlier, powder properties can be checked and noticed with particle size measurements, acting as fast quality controlling. Importance of this was noticed also by Mauer et al. [33] and in the review given by Yin et al. [15]. Particle morphology can have an influence on velocity as well. Especially, when comparing modelled and measured data, differences can be caused by morphology differences. Usually, in modelling, particles are expected to be spherical and have a certain particle size, but the practice has shown that there can be also some agglomerates, irregular or angular particles together with spherical ones [34]. Therefore, if powder is measured online from the spray jet, the particle size, powder quality, and powder feeding conditions can be checked prior spraying.

\subsection{Cold-Sprayed Al6061 Coatings-Structural Properties}

Coatings were sprayed with five selected spray parameters. The parameters resulting in the lowest particle velocities were left out from the coating characterization since high velocity assisted the higher coating quality. High temperature with low, medium, and high pressure as well as medium pressure with low, medium, and high temperature were selected for coating production. This way, we can investigate the effect of particle velocities on coating properties. First, coating thicknesses were evaluated as an indicator for deposition efficiency. Particle velocities had a noticeable effect on the coating thicknesses, as presented in Figure 6. Relative thickness was represented per one coating layer and it was normalized with the traverse speed. When spraying with high temperature, increment of coating thickness was $+13 \%$ when comparing medium pressure to low pressure and $+45 \%$ with high pressure compared to low pressure. The effect of temperature on the coating thickness was stronger than the effect of pressure. Coating thickness sprayed with medium pressure and medium temperature was $+65 \%$ higher compared to the coating thickness sprayed with medium pressure and low temperature. Thickness increased $+54 \%$ when sprayed with medium pressure and high 
temperature compared to medium pressure and $+154 \%$ compared to the low temperature. Higher gas temperature assisted thermal softening of the particles, which, in turn, increased the particle deformation $[7,10,12]$. In addition to this, heating increased the adherence. Also, with higher pressure and temperature of the gas, higher particle velocity is achieved, which, in turn, increases the deposition efficiency [35]. Therefore, it could be said that the deposition efficiency increased in this study as well while comparing the coating thicknesses. In all the cases, coating thickness increased with increasing spray parameters. This indicates that we are in the suitable spray window area with these selected spray parameters. If temperature or pressure is increased even more, there will come the limit and above this, thickness starts to decrease, as shown Schmidt et al. [13]. This was due to the erosion by the particles that started to play a role during the impacts [13].
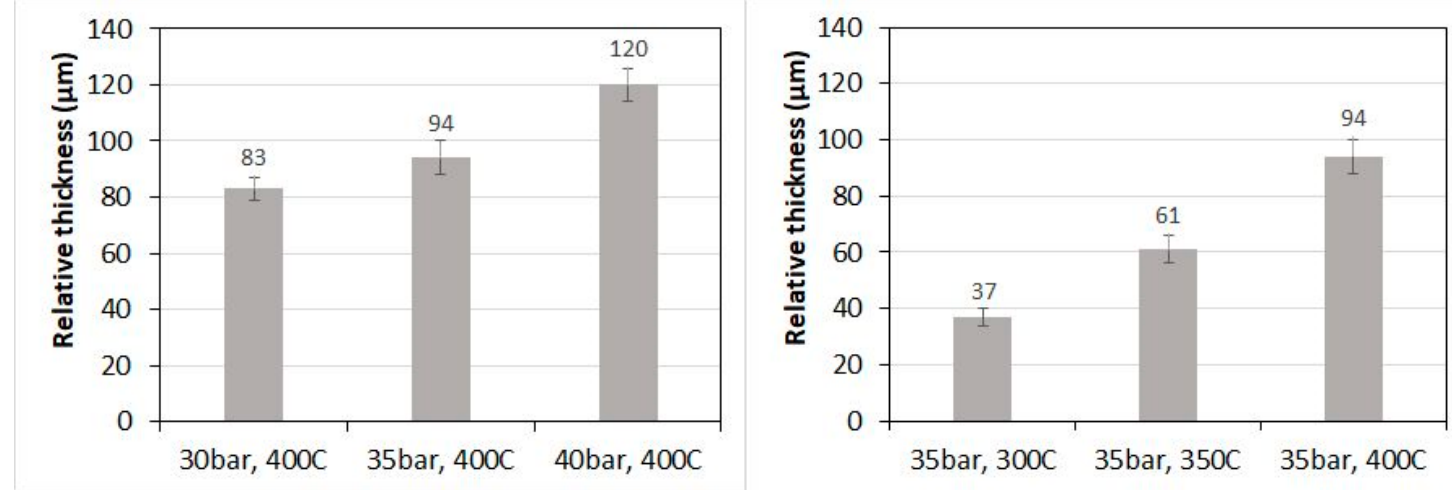

Figure 6. Relative thicknesses of the cold-sprayed Al6061 coatings sprayed with different spray parameters.

Cold-sprayed Al6061 coating structures sprayed with high temperature $\left(400^{\circ} \mathrm{C}\right)$ and with different pressures (30, 35, and 40 bar) are presented in Figure 7 . All the coatings are relatively dense by microscopic analysis and well adhered to the substrate. Particle velocity was higher than critical velocity for aluminum (620-660 m/s [13]) and therefore, coating structures were dense, and coating was well deformed. However, by increasing pressure, higher deformation can be achieved. This was seen as a purer structure sprayed with 40 bar and $400{ }^{\circ} \mathrm{C}$ (Figure $7 \mathrm{c}$ ), compared to structure sprayed with 30 bar and $400{ }^{\circ} \mathrm{C}$ (Figure 7a). In the latter case, some open particle boundaries were detected due to less deformed particles and more oxidized particle boundaries that were not destroyed during the particle impacts. Also, Cavaliere and Sivello [5] have concluded that with the nonoptimal spray conditions, adhesion of the cold-sprayed Al-based coatings was weaker and the structure included more pores, which, in turn, affected the fatigue resistance of the coatings. In general, high quality coatings had better fatigue resistance [5]. 


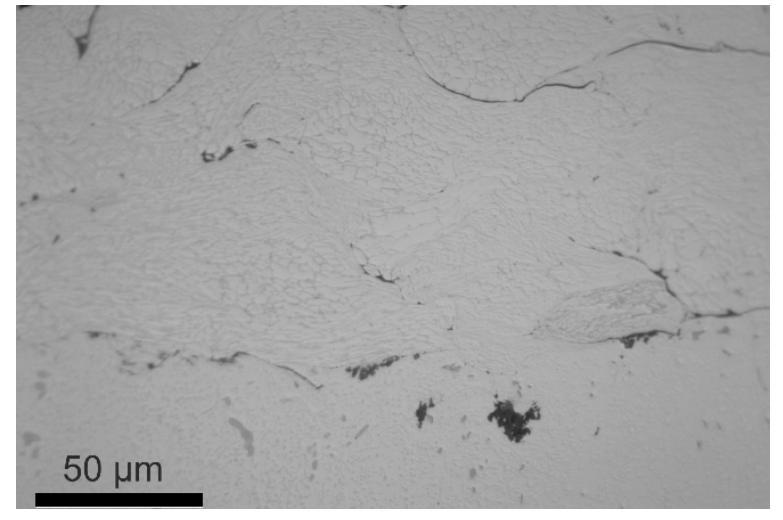

(a)

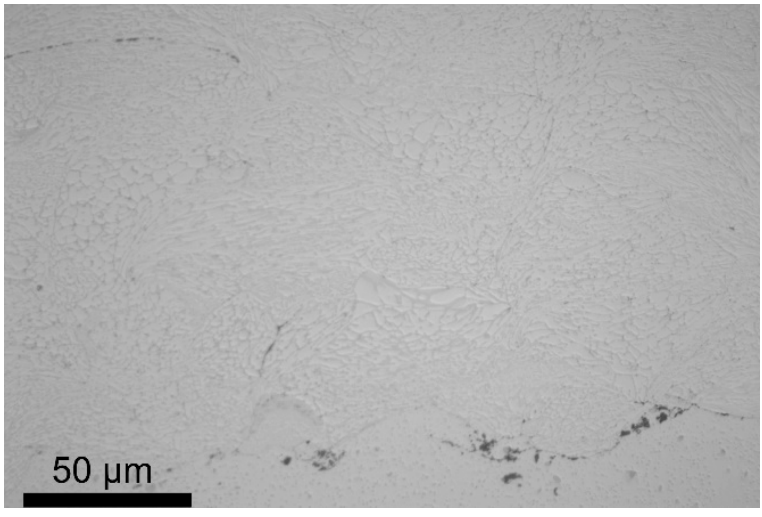

(b)

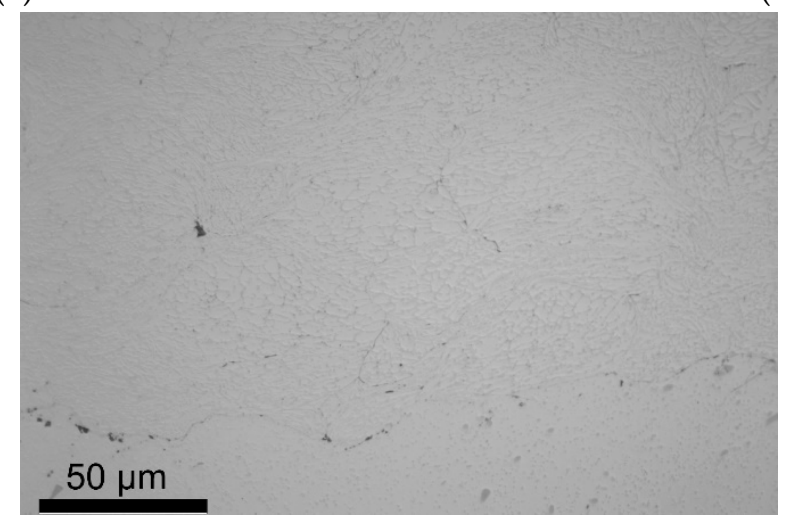

(c)

Figure 7. Structure and interface between CS Al6061 coating and Al substrate sprayed with different spray parameters (a) $30 \mathrm{bar}, 400{ }^{\circ} \mathrm{C}$, (b) $35 \mathrm{bar}, 40{ }^{\circ} \mathrm{C}$, and (c) $40 \mathrm{bar}, 400{ }^{\circ} \mathrm{C}$. LM images.

In cold spraying, powder particles experience high plastic deformation during the impacts. Bonding of particles and substrate, and particles together, is based on this plastic deformation and adiabatic shear instabilities at the interface. Furthermore, during the particle impacts, coming particles densify the previous particle structures by hammering or tamping them. [11] Sometimes, there is a porous top layer in the cold-sprayed coatings because the hammering effect of next particles is missing. However, this can be diminished by increasing particle velocity by increasing temperature and pressure [36]. Dense microstructures of cold-sprayed Al6061 coatings are also visual in FESEM images, Figure 8. Particles were highly deformed and only some open particle boundaries can be detected with the coatings sprayed with low or medium temperatures and pressures. Cold-sprayed Al6061 coating sprayed with the high temperature $\left(400^{\circ} \mathrm{C}\right)$ and pressure $(40 \mathrm{bar})$ was very dense and particles were extensively deformed. Cross-points between particles have been highlighted with the circles in Figure 8a-e, and especially, the cross-point between highly deformed particles has been marked in Figure 8e. This illustrates high deformation and bonding, which in turn, reflect the high-quality coating structure.

Here, cold-sprayed Al6061 coatings confirmed that higher particle velocity assisted the higher coating quality by means of denseness and purity. High particle velocity led to high deformation, which, in turn, led to tight bonding and removal of original powder particle oxide layers during the impacts [10]. If we compare the structures in Figure 8a,e, the difference was detectable while comparing the parts highlighted with the circles. Mean particle velocity difference between these coatings was $70 \mathrm{~m} / \mathrm{s}$ (Figure 4), which, in turn, had significant influence in this study. 


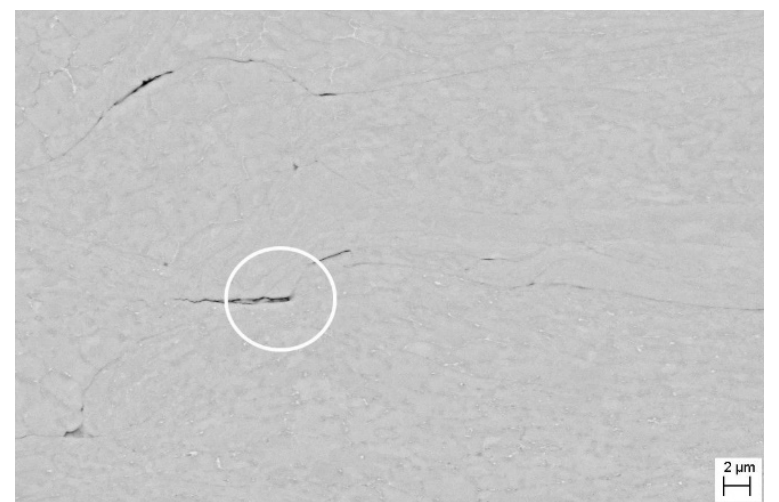

(a)

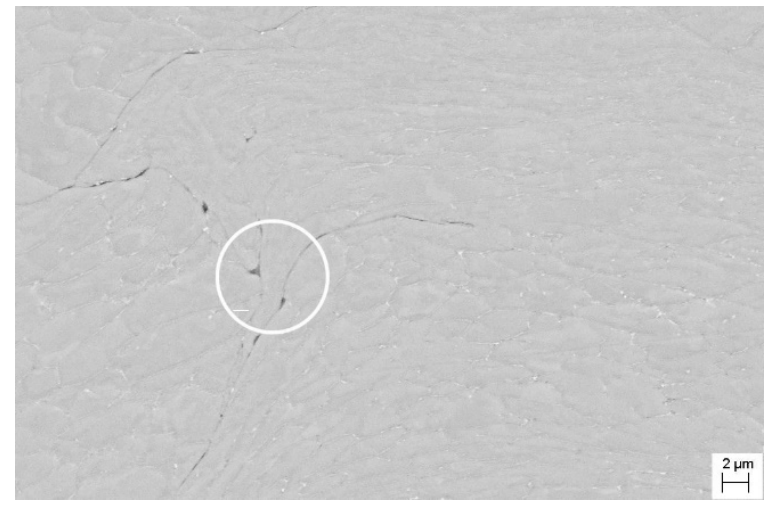

(c)

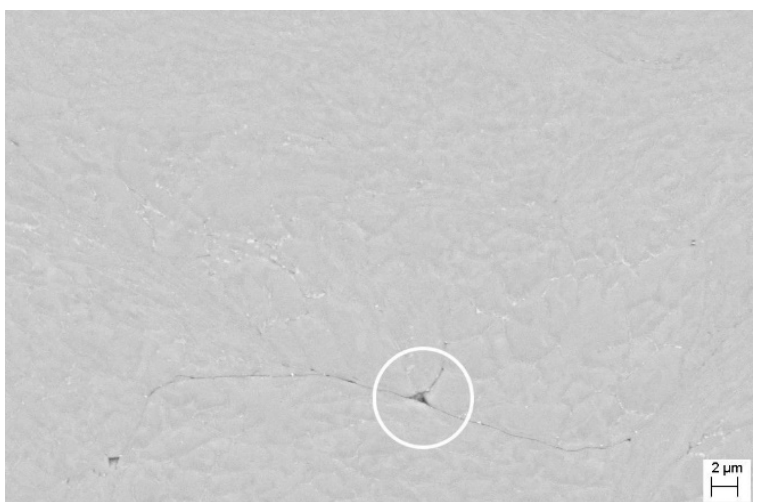

(b)

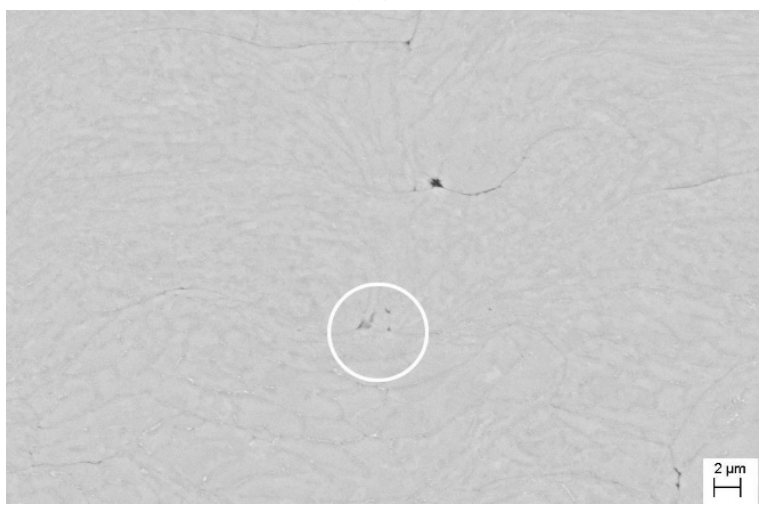

(d)

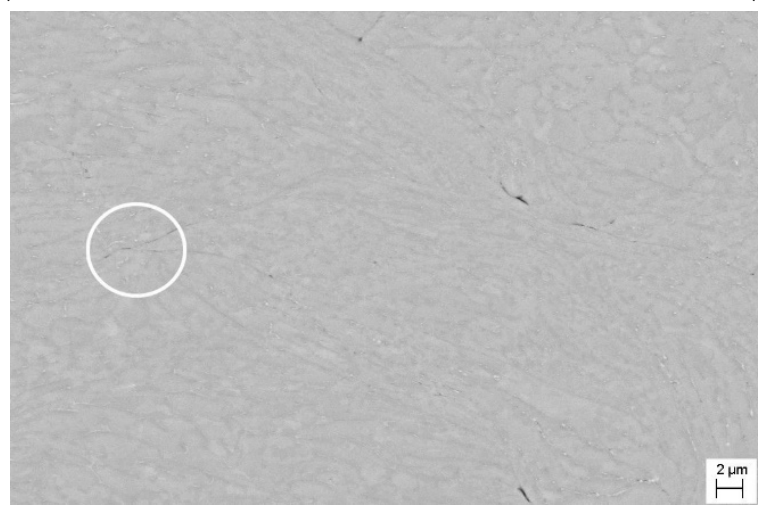

(e)

Figure 8. Microstructures of cold-sprayed Al6061 coatings sprayed with different spray parameters (a) 35 bar; $300{ }^{\circ} \mathrm{C}$, (b) 35 bar; $350{ }^{\circ} \mathrm{C}$, (c) 30 bar; $400{ }^{\circ} \mathrm{C}$, (d) 35 bar; $400{ }^{\circ} \mathrm{C}$, and (e) 40 bar; $400{ }^{\circ} \mathrm{C}$. Circles show the cross-points between particles. FESEM images.

\subsection{Cold-Sprayed Al6061 Coatings-Mechanical Properties}

Spray parameters did not have detectable effects on coating hardness. All cold-sprayed Al6061 coatings in this study had a hardness around $96 \mathrm{HV}_{0.1}$, Figure 9. They are much harder than A16061-O fully annealed bulk material (Brinell hardness 30 [30], $40 \mathrm{HV}$ ), which is annealed in the temperature of $415{ }^{\circ} \mathrm{C}$ [37]. Typically, $\mathrm{Al}$ alloys are solution heat-treated in order to increase their mechanical properties. Hardness of Al6061-T4 bulk material, which is solution heat-treated and naturally aged [37], is 65 of Brinell hardness [30] (74 HV) and furthermore, hardness of Al6061-T6, which is solution heat treated and artificially overaged [37], is 95 of Brinell hardness [30] (111 HV). Hardness of cold-sprayed Al6061 coatings were much higher than Al6061-T4 but slightly lower than A16061-T6, meaning that during the cold spray processing, high mechanical properties can be achieved without the need of 
an addition post-treatment such as annealing. Gavras et al. [1] have produced Al6061 coatings with cold spraying by using He as the process gas. They achieved hardness for the as-sprayed coating as high as $105 \mathrm{HV}_{100}$. Hardness was slightly higher than in this study. Furthermore, annealing increased ductility and diffusion occurred at the particle boundaries. Similarity, resistance to fatigue crack growth was increased with annealing [1]. In our study, the coating hardnesses were at a similar level as they were in the previous study, where the coatings were sprayed with laser-assisted cold spraying. This indicates possible recovering already during the spraying with the higher process temperature [2]. The coating hardness was $\sim 106 \mathrm{HV}_{0.3}$, which was slightly higher than the hardness in this study. Earlier, the medium-pressure cold spray system with the pressure of 27.5 bar and the temperature of $350{ }^{\circ} \mathrm{C}$ was used as the process parameters. Unfortunately, we did not measure particle velocities in the previous study, but we expect that they were lower than in this study due to the lower spray parameter values used.

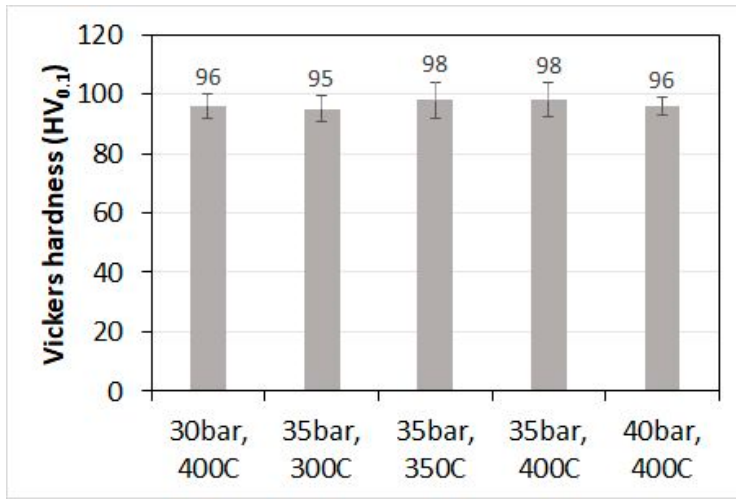

Figure 9. Vickers hardness $\left(\mathrm{HV}_{0.1}\right)$ and standard deviations of the cold-sprayed Al6061 coatings sprayed with different spray parameters.

Adhesion of the cold-sprayed Al6061 coatings was tested with the tensile pull tests. Substrate material together with the process parameters influenced on the bonding. Earlier, it was noticed that coating adhesion was higher on soft metal substrate compared to harder substrate material [2,38]. Similarly here, adhesion strength of the cold-sprayed Al6061 coatings were higher on the Al substrate $(\sim 35 \mathrm{MPa})$ compared to those on the low carbon steel substrate ( 20 MPa), Figure 10. In all cases, fracture occurred in the coating-substrate interface, indicating adhesive failure. It seemed that certain pressure level is needed for good bonding. In this study, pressure of 35 bar or 40 bar was needed for spraying Al6061 material with cold spraying, which meant that particle velocity was higher than $700 \mathrm{~m} / \mathrm{s}$. Higher particle velocity was beneficial for the good adhesion and cohesion. Bonding was improved with high particle velocities due to the higher deformation and thermal softening caused by increased impact temperature [5].

Adhesion of the cold-sprayed coatings can be improved by spray parameter optimization, or with advanced substrate surface pre-treatments, e.g., laser texturing [39] or by using assisting spray processes, e.g., laser-assisted cold spraying [2,3] or by heat treatments as post-treatment [40]. While spraying $\mathrm{Al}$ alloy material by using cold spray processing with the pressure of 30 bar and heating of $500{ }^{\circ} \mathrm{C}$, Kromer et al. [37] achieved adhesion bond strengths for $\mathrm{Al}$ alloy coatings on grit-blasted, and two laser textured $\mathrm{Al}$ alloy substrates as 20,40 , and $50 \mathrm{MPa}$, respectively. It shows that with the best spray parameter combination in our study, we can achieve the coating adhesion close to the level that can be achieved with laser texturing. Our previous results [2] with a medium-pressure cold spray system operated at 27.5 bar and $350{ }^{\circ} \mathrm{C}$ achieved bond strengths of 27 and $9 \mathrm{MPa}$ for $\mathrm{Al}$ and steel substrates, respectively. Furthermore, the same system with an additional laser assistance produced coatings with bond strengths of $48 \mathrm{MPa}$ for $\mathrm{Al}$ and $35 \mathrm{MPa}$ for $\mathrm{Al}$ and steel substrates, respectively. 
The results of the present study in Figure 10 show that even higher adhesion can be achieved with the advanced cold spray system using the optimized process parameters.

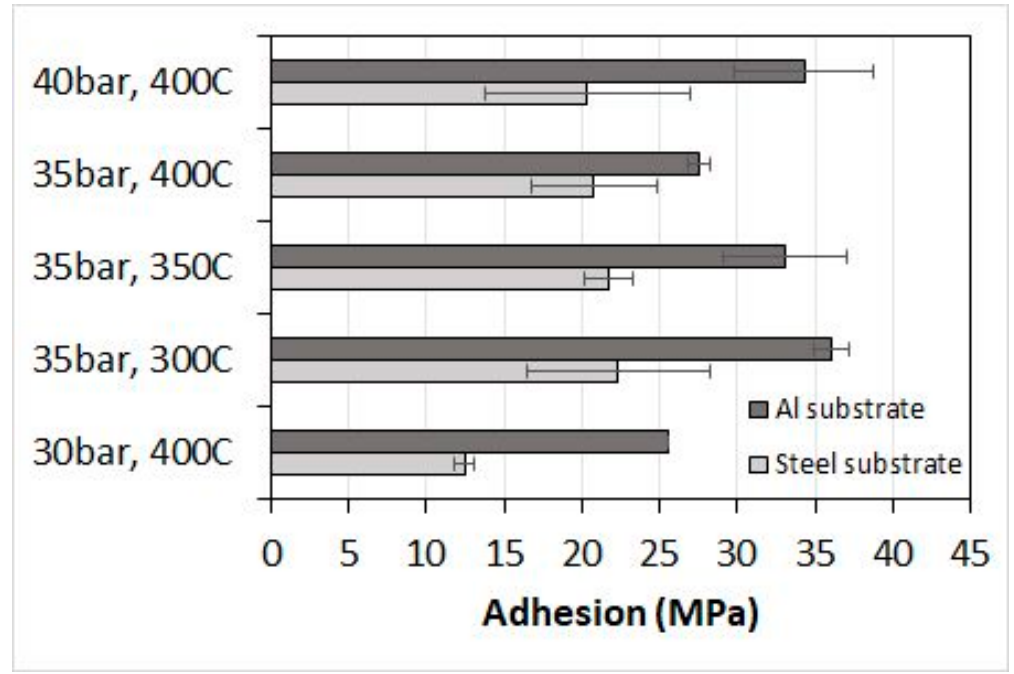

Figure 10. Adhesion strengths of the cold-sprayed Al6061 coatings sprayed with different spray parameters on grit-blasted $\mathrm{Al}$ and steel substrates.

\section{Conclusions}

Process optimization for cold-sprayed Al6061 powder was done by using online monitoring technique together with coating characterization. As already known, particle velocity has an important role in cold spraying for successful and high-performance coating production. Therefore, here we analyzed the influence of spray parameters on the particle mean velocity and its connections to the coating structure and properties. Particle velocity increased with increased pressure and gas temperature used. In addition, novel $2 \mathrm{D}$ velocity scan maps with the information about particle amounts connected to their velocities in the spray jet. This supported quality controlling and indicated more efficiently the powder and spray conditions. In this study, powder conditions were controlled with 2D velocity scan maps and particle size measurements, and they supported optimal spray conditions from the technical point of view. The nozzle worked well and powder agglomeration was not observed, indicating appropriate cold-spray processing.

Cold-sprayed Al6061 coatings were relatively dense, but by using higher particle velocities using higher process parameters resulted in even denser and more deformed coating structures were achieved. In this study, relatively high process parameters (high pressures: $30-40 \mathrm{bar}$, and high gas temperature for $\mathrm{Al}$ alloys: $300-400{ }^{\circ} \mathrm{C}$ ) were used due to the wide spray parameter possibilities available in the high-pressure cold spray equipment. Process parameters did not affect the coating hardness in this study. Hardnesses were in the same level, $96 \mathrm{HV}$. On the other hand, adhesion of the coating was slightly influenced by process parameters. Both high pressure and high gas temperature was needed for higher coating adhesion, resulting from the higher particle velocities, and therefore, higher impact conditions during the spraying. Next, our research will focus on cold spraying of thick structures utilizing the knowledge achieved in this study. For this, accurate quality control by online monitoring plays key role together with optimized process conditions used while going towards cold spray additive manufacturing.

Author Contributions: Conceptualization, investigation and writing-original draft, H.K.; investigation and editing, J.L.; investigation, editing D.M., G.P., and F.M. All authors have read and agreed to the published version of the manuscript.

Funding: This research received no external funding. 
Acknowledgments: Authors would like to thank Jarkko Lehti of Tampere University, Thermal Spray Center Finland (TSCF), Tampere, Finland for spraying the coatings and Jarmo Laakso of Tampere University, Tampere, Finland for SEM characterization of the powder. This work made use of Tampere Microscope Center facilities at Tampere University, Tampere, Finland. Also, The Scientific Advisory Board for Defense (MATINE), Finland is acknowledged.

Conflicts of Interest: The authors declare no conflicts of interest.

\section{References}

1. Gavras, A.G.; Lados, D.; Champagne, V.K.; Warren, R. Effects of processing on microstructure evolution and fatigue crack growth mechanisms in cold-spray 6061 aluminum alloy. Int. J. Fatigue 2018, 110, 49-62. [CrossRef]

2. Koivuluoto, H.; Milanti, A.; Bolelli, G.; Latokartano, J.; Pulci, G.; Vihinen, J.; Lusvarghi, L.; Vuoristo, P.; Marra, F. Structures and Properties of Laser-Assisted Cold-Sprayed Aluminum Coatings. Mater. Sci. Forum 2016, 879, 984-989. [CrossRef]

3. Koivuluoto, H.; Kiilakoski, J.; Costil, S. Effect of Laser Texturing of Substrate Surface on Coating Properties of Cold-Sprayed and Laser-Assisted Cold-Sprayed Aluminum Coatings. In Proceedings of the International Thermal Spray Conference 2019, Yokohama, Japan, 26-29 May 2019; pp. 810-815.

4. White, B.; Story, W.; Brewer, L.; Jordon, J.B. Fatigue behaviour of fastener holes in high-strength aluminium plates repaired by cold spray deposition. Fatigue Fract. Eng. Mater. Struct. 2019, 43, 317-329. [CrossRef]

5. Cavaliere, P.; Silvello, A. Crack Repair in Aerospace Aluminum Alloy Panels by Cold Spray. J. Therm. Spray Technol. 2017, 26, 661-670. [CrossRef]

6. Papyrin, A.; Kosarev, V.; Klinkov, S.; Alkhimov, A.; Fomin, V.M. (Eds.) Cold Spray Technology, 1st ed.; Elsevier Science: London, UK, 2006; p. 336.

7. Cavaliere, P. (Ed.) Cold-Spray Coatings: Recent Trends and Future Perspectives; Springer International Publishing: Berlin, Germany, 2018; p. 570.

8. Villafuerte, J. (Ed.) Modern Cold Spray: Materials, Process, and Applications; Springer International Publishing: Berlin, Germany, 2015; p. 429.

9. Maev, R.; Leshchynsky, V. Introduction to Low Pressure Gas Dynamic Spray: Physics and Technology; Wiley: Hoboken, NJ, USA, 2009; p. 234.

10. Champagne, V.K. (Ed.) The Cold Spray Materials Deposition Process: Fundamentals and Applications; Woodhead Publishing Limited: Cambridge, UK, 2007; p. 376.

11. Luo, X.-T.; Li, C.-X.; Shang, F.; Yang, G.-J.; Wang, Y.-Y.; Li, C. High velocity impact induced microstructure evolution during deposition of cold spray coatings: A review. Surf. Coat. Technol. 2014, 254, 11-20. [CrossRef]

12. Assadi, H.; Kreye, H.; Gärtner, F.; Klassen, T. Cold spraying-A materials perspective. Acta Mater. 2016, 116, 382-407. [CrossRef]

13. Schmidt, T.; Assadi, H.; Gärtner, F.; Richter, H.; Stoltenhoff, T.; Kreye, H.; Klassen, T.; Gaertner, F. From Particle Acceleration to Impact and Bonding in Cold Spraying. J. Therm. Spray Technol. 2009, 18, 794-808. [CrossRef]

14. Schmidt, T.; Gärtner, F.; Assadi, H.; Kreye, H.; Gaertner, F. Development of a generalized parameter window for cold spray deposition. Acta Mater. 2006, 54, 729-742. [CrossRef]

15. Yin, S.; Meyer, M.; Li, W.; Liao, H.; Lupoi, R. Gas Flow, Particle Acceleration, and Heat Transfer in Cold Spray: A review. J. Therm. Spray Technol. 2016, 25, 874-896. [CrossRef]

16. Sova, A.; Smurov, I.Y.; Doubenskaia, M.; Petrovskiy, P. Deposition of aluminum powder by cold spray micronozzle. Int. J. Adv. Manuf. Technol. 2017, 95, 3745-3752. [CrossRef]

17. Gilmore, D.; Dykhuizen, R.; Neiser, R.; Roemer, T.; Smith, M. Particle Velocity and Deposition Efficiency in the Cold Spray Process. J. Therm. Spray Technol. 1999, 8, 576-582. [CrossRef]

18. Champagne, V.K.; Helfritch, D.J.; Dinavahi, S.P.G.; Leyman, P.F. Theoretical and Experimental Particle Velocity in Cold Spray. J. Therm. Spray Technol. 2010, 20, 425-431. [CrossRef]

19. Fukanuma, H.; Ohno, N.; Sun, B.; Huang, R. In-flight particle velocity measurements with DPV-2000 in cold spray. Surf. Coat. Technol. 2006, 201, 1935-1941. [CrossRef]

20. Suo, X.; Yin, S.; Planche, M.-P.; Liu, T.; Liao, H. Strong effect of carrier gas species on particle velocity during cold spray processes. Surf. Coat. Technol. 2015, 268, 90-93. [CrossRef] 
21. Ajdelsztajn, L.; Jodoin, B.; Richer, P.; Sansoucy, E.; Lavernia, E. Cold Gas Dynamic Spraying of Iron-Base Amorphous Alloy. J. Therm. Spray Technol. 2006, 15, 495-500. [CrossRef]

22. Pardhasaradhi, S.P.; Venkatachalapathy, V.; Joshi, S.; Govindan, S. Optical Diagnostics Study of Gas Particle Transport Phenomena in Cold Gas Dynamic Spraying and Comparison with Model Predictions. J. Therm. Spray Technol. 2008, 17, 551-563. [CrossRef]

23. Wu, J.; Fang, H.; Yoon, S.; Kim, H.; Lee, C. Measurement of particle velocity and characterization of deposition in aluminum alloy kinetic spraying process. Appl. Surf. Sci. 2005, 252, 1368-1377. [CrossRef]

24. Sova, A.; Okunkova, A.A.; Grigoriev, S.; Smurov, I. Velocity of the Particles Accelerated by a Cold Spray Micronozzle: Experimental Measurements and Numerical Simulation. J. Therm. Spray Technol. 2012, 22, 75-80. [CrossRef]

25. Meyer, M.; Yin, S.; Lupoi, R. Particle In-Flight Velocity and Dispersion Measurements at Increasing Particle Feed Rates in Cold Spray. J. Therm. Spray Technol. 2016, 26, 60-70. [CrossRef]

26. Koivuluoto, H.; Matikainen, V.; Larjo, J.; Vuoristo, P. Novel Online Diagnostic Analysis for In-Flight Particle Properties in Cold Spraying. J. Therm. Spray Technol. 2018, 27, 423-432. [CrossRef]

27. Sova, A.; Doubenskaia, M.; Petrovskiy, P.; Smurov, I.Y. Visualization of particle jet in cold spray by infrared camera: Feasibility tests. Int. J. Adv. Manuf. Technol. 2017, 95, 3057-3063. [CrossRef]

28. Jodoin, B.; Raletz, F.; Vardelle, M. Cold spray modeling and validation using an optical diagnostic method. Surf. Coat. Technol. 2006, 200, 4424-4432. [CrossRef]

29. Silvello, A.; Cavaliere, P.; Albaladejo, V.; Martos, A.; Dosta, S.; Cano, I.G. Powder Properties and Processing Conditions Affecting Cold Spray Deposition. Coatings 2020, 10, 91. [CrossRef]

30. Anderson, K.; Weritz, J.; Kaufman, J.G. (Eds.) 6061 and Alclad 6061: General Structural Alloy, Properties of Selection of Aluminium Alloys, ASM Handbook; ASM International: Geauga County, OH, USA, 2019; pp. 388-393.

31. ASTM C633, Standard Test Method for Adhesion or Cohesion Strength of Thermal Spray Coatings; ASTM: West Conshohocken, PA, USA, 2001; pp. 1-7.

32. Raoelison, R.; Xie, Y.; Sapanathan, T.; Planche, M.-P.; Kromer, R.; Costil, S.; Langlade, C. Cold gas dynamic spray technology: A comprehensive review of processing conditions for various technological developments till to date. Addit. Manuf. 2018, 19, 134-159. [CrossRef]

33. Alkhimov, A.; Kosarev, V.; Klinkov, S. The Features of Cold Spray Nozzle Design. J. Therm. Spray Technol. 2001, 10, 375-381. [CrossRef]

34. Mauer, G.; Singh, R.; Rauwald, K.-H.; Schrüfer, S.; Wilson, S.; Vaßen, R. Diagnostics of Cold-Sprayed Particle Velocities Approaching Critical Deposition Conditions. J. Therm. Spray Technol. 2017, 26, 1423-1433. [CrossRef]

35. Li, Y.; Wei, Y.; Luo, X.; Li, C.; Ma, N. Correlating particle impact condition with microstructure and properties of the cold-sprayed metallic deposits. J. Mater. Sci. Technol. 2020, 40, 185-195. [CrossRef]

36. Koivuluoto, H.; Vuoristo, P. Structural Analysis of Cold-Sprayed Nickel-Based Metallic and Metallic-Ceramic Coatings. J. Therm. Spray Technol. 2010, 19, 975-989. [CrossRef]

37. Hirsch, J. Annealing of Aluminum and Its Alloys, Heat Treating of Nonferrous Alloys, ASM Handbook; ASM International: Geauga County, OH, USA, 2016; pp. 137-147.

38. Stoltenhoff, T.; Borchers, C.; Gärtner, F.; Kreye, H. Microstructures and key properties of cold-sprayed and thermally sprayed copper coatings. Surf. Coat. Technol. 2006, 200, 4947-4960. [CrossRef]

39. Kromer, R.; Costil, S.; Verdy, C.; Gojon, S.; Liao, H. Laser surface texturing to enhance adhesion bond strength of spray coatings-Cold spraying, wire-arc spraying, and atmospheric plasma spraying. Surf. Coat. Technol. 2018, 352, 642-653. [CrossRef]

40. Rokni, M.; Widener, C.A.; Ozdemir, O.; Crawford, G. Microstructure and mechanical properties of cold sprayed $6061 \mathrm{Al}$ in As-sprayed and heat treated condition. Surf. Coat. Technol. 2017, 309, 641-650. [CrossRef]

(C) 2020 by the authors. Licensee MDPI, Basel, Switzerland. This article is an open access article distributed under the terms and conditions of the Creative Commons Attribution (CC BY) license (http://creativecommons.org/licenses/by/4.0/). 